\title{
Endothelium: from cellophane to orchestral maestro
}

\author{
Ralph L. Nachman \\ Weill Cornell Medical College, New York, New York, USA.
}

\begin{abstract}
Endothelial cells from human umbilical veins were first cultured nearly four decades ago, initiating explosive growth in research in vascular biology and leading to major insights into angiogenesis, vasculogenesis, and tumor biology. Recent studies now promise to open new horizons in regenerative medicine as well as organ engineering.
\end{abstract}

In 1973, the JCI published two back-toback papers from my laboratory, reporting the first successful in vitro culture of endothelial cells from human umbilical veins $(1,2)$. Over the last four decades, these papers have been listed among the most highly cited articles in the journal's history (7,024 times as of this writing). In 2004 , as part of the 80th anniversary of the JCI, Eric Jaffe and I reviewed the history of our somewhat circuitous and serendipitous journey leading to this discovery (3).

The project was driven by our realization that the endothelial cell was the key to understanding how the vascular wall worked in health and disease. We had learned much from studying the platelet, but it was clear that the orchestral leader in hemostasis had to be the endothelial cell. This sounds obvious now, but the prevailing view at the time was that endothelium should be regarded as analogous to the inner tube of a tire, what Lord Florey referred to as a "sheet of nucleated cellophane" $(4,5)$. How times have changed!

The two papers had a catalytic effect on the growth and development of the field of vascular biology by developing a workable approach to the endothelial cell using an available human tissue source (umbilical veins) to grow a pure population of cells using a definitive marker, vWF. The next year, Gimbrone, Cotran, and Folkman (6) corroborated the system, and the flood gates began to open; the number of related publications has grown dramatically since then.

The ability to culture the endothelial cell allowed researchers to understand and appreciate its role as the director of

Conflict of interest: The author has declared that no conflict of interest exists.

Citation for this article: J Clin Invest. 2012; 122(3):796-797. doi:10.1172/JCI62589. thromboregulation with important clinical implications. A number of inherited thrombophilic states were shown to be associated with abnormalities of inherent constitutive endothelial nonthrombogenic functions (Figure 1). In addition, the endothelium at rest maintains blood fluidity by cross-talk with platelets. In the activated nonconstitutive state, the endothelium plays a major role in the pathophysiology of conditions such as cancer and inflammation. In some activated conditions, including atherosclerotic disease and thrombosis, the endothelial cell synthesizes membrane-associated adhesion molecules including vWF, generates membrane assembly of modulators of thrombin gen-

eration, and at the same time downregulates the anticoagulant, antifibrinolytic thrombomodulin system (7).

The endothelial cell and the megakaryocyte are the only cells that synthesize vWF, strengthening the concept of a functional endothelial cell-megakaryocyte axis. The orchestration of platelet and endothelial secretion and release of intracellular constituents significantly influences the behavior of the vascular bed. Platelet production is supported by intravascular proplatelet formation after direct contact between marrow sinusoidal endothelium and megakaryocytes. There is abundant molecular cross-talk between these two cell systems, conversations that in steady state constitute a mechanism for the biochemical nourishment and stabilization of the intact vessel wall.

The exciting studies performed over the last several decades on endothelial cell biology have lead to tremendous insights into

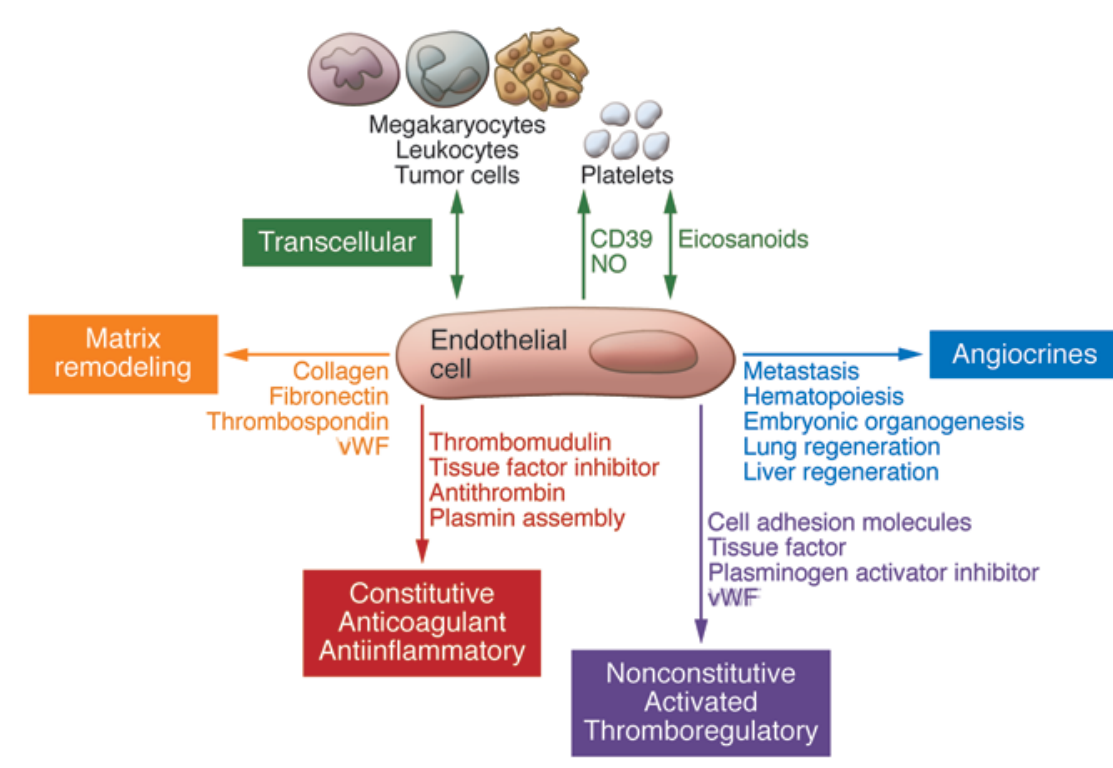

Figure 1

The endothelial cell symphony. The endothelial cells conduct luminal constitutive, anticoagulant, and antiinflammatory functions as well as nonconstitutive, activated, thromboregulatory activities. Furthermore, these cells secrete angiocrines, which orchestrate a number of different pathways, including embryonic organogenesis, hematopoiesis, metastasis, and lung and liver regeneration. 
the fundamental processes of angiogenesis and vasculogenesis, cancer research, and even our understanding of organ development and regeneration. Bone marrowderived endothelial progenitor cells have been shown to participate in adult vasculogenesis or new vessel formation by directly seeding preconditioned sites and acting in concert with hematopoietic precursors. Blocking this process interferes with tumor growth (8). In addition, endothelial cells in the marrow - via production of specific stem and progenitor cell trophogen factors (angiocrines) - directly control hematopoietic stem cell self renewal as well as differentiation (9). The recently recognized molecular heterogeneity of specialized vascular beds in different organs has highlighted the controlling influence of specific microenvironmental signals on organ function and development. Thus, organ-specific angiocrines promote hematopoiesis in the bone marrow as well as regeneration in the liver and lung $(10,11)$.

The future promises major new endothelial cell developments in regenerative medicine and eventually organ engineering. We have come a long way from our conception of endothelium as inert cellophane to our current appreciation of it as a cellular orchestral maestro.

Address correspondence to: Ralph L. Nachman, Weill Cornell Medical College, 1300 York Avenue, Box 305, New York, New York 10065, USA. Phone: 212.746.7723; Fax: 212.746.8343; E-mail: rlnachm@med. cornell.edu.

1. Jaffe ES, Nachman RL, Becker CG, Minick CR. Culture of human endothelial cells derived from umbilical veins: identification of morphologic and immunologic criteria. J Clin Invest. 1973;52(11):2745-2756

2. Jaffe ES, Hoyer LW, Nachman RL. Synthesis of antihemophilic factor antigen by cultured human endothelial cells. J Clin Invest. 1973;52(11):2757-2764.

3. Nachman RL, Jaffe EA. Endothelial cell culture: beginnings of modern vascular biology. J Clin Invest. 2004;114(8):1037-1040.

4. Florey L. The endothelial cell. $\mathrm{Br} \mathrm{Med} J .1966$; 2:487-490.

5. Kaiser L, Sparks HV. Endothelial cells. Not just cellophane wrapper. Arch Intern Med. 1987; 147(3):569-573.

6. Gimbrone MA, Cotran RS, Folkman J. Human vascular endothelial cells in culture. Growth and DNA synthesis. J Cell Biol. 1974;60(3):673-684.

7. Stern DM, Kaiser E, Naworth P. Regulation of the coagulation system by vascular endothelial cells. Haemostasis. 1988;18(4-6):202-214

8. Lyden D, et al. Impaired recruitment of bone-marrow-derived endothelial and hematopoietic precursor cells blocks tumor angiogenesis and growth. Nat Med. 2001;7(11):1194-1201.

9. Kobayashi $\mathrm{H}$, et al. Angiocrine factors from Aktactivated endothelial cells balance self-renewal and differentiation of haematopoietic stem cells. Nat Cell Biol. 2010;11(9):1046-1056.

10. Ding BS, et al. Inductive angiocrine signals from sinusoidal endothelium and required for liver regeneration. Nature. 2010;468(7321):310-315.

11. Ding BS, et al. Endothelial-derived angiocrine signals induce and sustain regenerative lung alveolarization. Cell. 2011;147(3):539-553. 